\title{
Fuzzy optimal portfolio selection based on multi-objective Mean-Variance-Skewness model by using NSGA-II algorithm
}

\author{
Sharareh ASHRAFZADEH ${ }^{1}$, Mehdi MORADZADEHFARD ${ }^{2, *}$, Fereydoun OHADI ${ }^{3}$ \\ ${ }^{1}$ Faculty of Management and Accounting, Islamic Azad University, Karaj branch, Iran \\ ${ }^{2}$ Faculty of Management and Accounting, Islamic Azad University, Karaj branch, Iran \\ ${ }^{3}$ Department of Industrial Engineering, Islamic Azad University, Karaj branch, Iran
}

\begin{abstract}
Constructing an optimal portfolio is a critical decision for investors. The classic portfolio models generally consider mean and variance of return criteria, which are mostly intended single objective and have been analyzed and studied under conditions of certainty. But in the real world problem of portfolio selection, is a multi-objective problem and in addition to the criteria of mean and variance of return, other criteria such as liquidity risk should also be considered. On the other hand, in practice we are faced with a vague inaccurate data and portfolio selection problem must be studied under conditions of fuzzy uncertainty. In this paper, we have developed a new fuzzy multi-objective programming model based on mean-variance-skewness model for optimal portfolio selection under fuzzy uncertainty. The objective functions include maximizing the expected return, maximizing skewness or the chance to gain expected returns and minimizing liquidity risk. Rates of return, rates of turnover, and the maximum number of types of stocks included in the portfolio have been considered as triangular fuzzy numbers. To solve this problem an elitist non-dominated sorting genetic algorithm (NSGA-II) has been developed. Vector evaluated genetic algorithm (VEGA) and non-dominated sorting genetic algorithm (NSGA) were used to compare and evaluate the performance of the proposed solving method. Finally, the results obtained from the algorithms output are compared and analyzed, which indicates a higher efficiency of the proposed solving method compared to other methods.
\end{abstract}

Keywords: portfolio selection problem, the mean-variance-skewness model, NSGA-II algorithm, multi-objective programming, fuzzy numbers

\section{Introduction}

The main goal of modeling portfolio is to help the investor in optimal portfolio selection according to his preferences and interests as well as the decision environment [1]. In 1950, Harry Markowitz provide a basic portfolio model that has become the basis for modern portfolio theory. He was the first to express the concept of the portfolio and diversification as a formal way. He showed quantitatively why and how portfolio diversification can reduce portfolio risk for an investor [2]. Markowitz mean-variance model is the most famous and most popular approach to the portfolio selection problem. The most outstanding point of interest in Markowitz model is to consider investment risk not only based on the standard deviation of returns of a stock but to consider the risk of the set of investment $[1,3]$.

The mean-variance model has several simplifying assumptions and several weaknesses, which leads to inefficiency in the real-world problems. The criterion of return variance in its high state has the behavior like low return which has undesirable behavior because the high return also contributes in increasing the variance. In fact, when the probability distribution of stock returns is asymmetric, the variance will be an inefficient measure of investment risk. To overcome the weakness of variance risk measure of Markowitz model, some researchers have begun to use other risk criteria to determine the risk of their portfolios [4]. One of the solutions proposed is the application of the criteria of return distribution skewness in optimal portfolio selection so that, mean-variance-skewness model was introduced $[5,6]$.

On the other hand, in most studies, the risk of an asset is identified as a random variable with a probability distribution of the returns. But in a real investment, returns of risky assets are usually in an uncertain economic environment and varies from time to time. So, since there are a number of uncertain parameters that are different from the random effects of financial markets, such as economics, politics, laws and regulations, people's subjective factors, etc., it is impossible for the investor to obtain accurate, probability distribution of returns of risky assets. In practice, people's subjective factors should be considered in the decision-making portfolios, and these factors are affecting financial markets. To get public opinion and uncertainty in the financial markets in terms of our calculations, fuzzy methods are generally more appropriate than probability methods. So it is valued to apply fuzzy set theory to evaluate the uncertainty in the financial markets $[4,5,7]$.

\footnotetext{
* Corresponding author: Mehdi Moradzadehfard

Email: moradzadehfard@kiau.ac.ir
} 
Also, disregarding the portfolio liquidity risk and transaction costs, modeling and solving portfolio selection problems for single-objective or converting a multi-objective portfolio selection model into a single-objective problem and solving it at a later stage, have led to models and methods that are far from investment issues in the real world and have lost their effectiveness [8,9].

Recently, some researchers have tried to reduce vulnerabilities of mean-variance Markowitz model. Ammar and Khalifa [10], in their article using fuzzy intervals, modeled the portfolio optimization in a manner that minimizes investment risk so that, return more than a specified amount will be determined. They solved their nonlinear programming model by using the Lagrange multipliers. The result is the allocation of funds to each investment considering the budget restrictions. Huang [11,12] defined fuzzy-semi-variance as the main variable of portfolio risk and accordingly, presented mean-semi-variance fuzzy model. Liu and Zhang [3] developed a multi-objective portfolio optimization model based on fuzzy mean-semi variance model for portfolio selection. They proposed two mean-semi-variance probability model with the real constraints. For solving proposed models a fuzzy multi-objective programming method is used to convert them into a single objective model and then a genetic algorithm has been designed to solve it. Khalifa and ZeinEldin [8] presented a fuzzy programming model for multi-objective portfolio selection model. They considered fuzzy numbers as objective function coefficients and proposed $\alpha$ cutting technique and simulated annealing meta-heuristic methods to solve the problem. Shah Mohammadi et al. [5] modeled a portfolio selection problem based on the mean-variance-skewness model, where stock returns are assumed to be fuzzy variables and to solve this nonlinear model, a genetic algorithm with the neural network was used. Shibt al-Hamdi et al. [13] used Genetic Algorithm NSGA-II in a deterministic multiobjective portfolio optimization problem and demonstrated this solution has higher efficiency compared to other methods for solving multi-objective problems. Delvi et al. [14] adopted a multi-objective genetic algorithm to select the optimal portfolio of facilities of Melli banks in Isfahan province. The results show that the optimal portfolio of facilities that has been achieved by the multi-objective genetic algorithm is different from the bank's current portfolio and is covering different policies and restrictions prevailing lending and is more efficient.

The aim of this paper is to develop a new fuzzy multi-objective programming model based on Mean-varianceskewness model for optimal portfolio selection under conditions of fuzzy uncertainty. The objective functions include maximizing the expected return, maximizing skewness or a chance to obtain expected returns and minimizing liquidity risk. Rates of return and rates of turnover, and the maximum number of types of stocks included in the portfolio have been considered for triangular fuzzy numbers. To solve this problem, a genetic algorithm NSGA-II has been developed and its performance was compared and analyzed by genetic algorithms VEGA and NSGA. Thus simplifying assumptions in modeling and solving portfolio selection problem is reduced and proposed model is more efficient in real-world problems.

The paper is organized as follows. In section 2 the exact definition of the problem and its mathematical model have been presented. The methodology of problem-solving and multi-objective genetic algorithms have been developed and described in Section 3. In section 4, the computational results and comparisons have been expressed in order to demonstrate the applicability and efficiency of the model and the solving method. The conclusions and directions of future work are provided in Section 5.

\section{Problem Formulation}

\section{1 Expected value, variance and skewness of fuzzy variables}

Professor Lotfi A. Zadeh in 1965 for the first time with the introduction of fuzzy set theory provided preparations of inaccurate information modeling and approximate reasoning with mathematical equations that in itself brought enormous changes in mathematics and classical logic [7,8]. The possibility theory is one of the most important issues of fuzzy set theory which is used to deal with most phenomena of the real world in which there is uncertainty. This theory is somewhat similar to classical probability theory in crisp sets [5]. In order to eliminate the weaknesses of the possibility theory, Liu and Liu in 2002 proposed the credibility theory as a rival to the possibility theory. According to this theory, the purpose of the credibility of a fuzzy event is the chance of a fuzzy event [15].

Suppose that $\tilde{r}$ is a fuzzy variable with membership function $\mu$, and $k$ and $u$ are real numbers. Then the possibility and the necessity of a fuzzy event are obtained respectively from the equations (1) and (2).

$$
\begin{gathered}
\operatorname{pos}\{\tilde{r} \geq k\}=\operatorname{Sup} \mu(u) \quad, \quad u \geq k \\
\operatorname{Nes}\{\tilde{r} \geq k\}=1-\operatorname{pos}\{\tilde{r}<k\}=1-\underset{u<k}{\operatorname{Sup}} \mu(u)
\end{gathered}
$$

The credibility of a fuzzy event is equal to the arithmetic mean value of the possibility and the necessity of a fuzzy event and its value is calculated from equation (3).

$$
\operatorname{Cr}\{\tilde{r} \geq k\}=\frac{1}{2}(\operatorname{pos}\{\tilde{r} \geq k\}+\operatorname{Nes}\{\tilde{r} \geq k\})
$$


As a result of the expected value of a fuzzy variable of $\tilde{r}$ is obtained from the equation (4).

$$
E(\tilde{r})=\int_{0}^{\infty} C r\{\tilde{r}>k\} d k-\int_{-\infty}^{0} C r\{\tilde{r} \leq k\} d k
$$

With the expected value, variance and skewness of fuzzy variable $\tilde{r}$ can be calculated by the equations (5) and (6).

$$
\begin{gathered}
\operatorname{Var}(\tilde{r})=E\left[(\tilde{r}-E(\tilde{r}))^{2}\right] \\
\operatorname{Sk}(\tilde{r})=E\left[(\tilde{r}-E(\tilde{r}))^{3}\right]
\end{gathered}
$$

If $\tilde{r}=(a, b, c)$ is a triangular fuzzy variable (Fig. 1.), membership function and its credibility will be as the equations (7) and (8) [15].

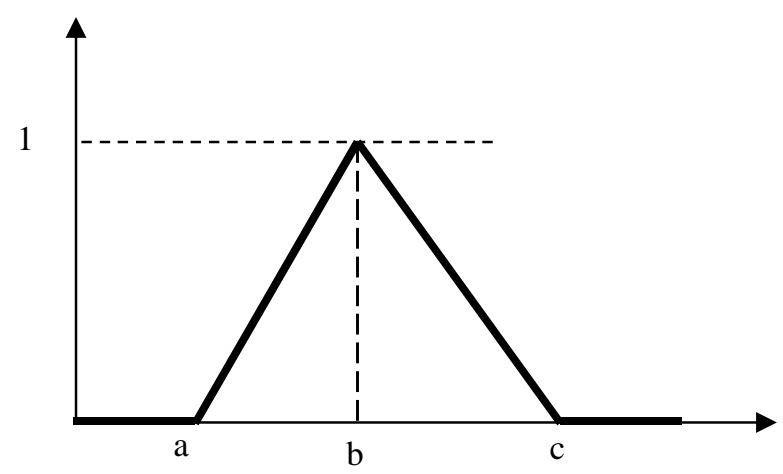

Fig. 1: A triangular fuzzy variable

$$
\begin{gathered}
\mu(k)= \begin{cases}\frac{k-a}{b-a} & a \leq k \leq b \\
\frac{c-k}{c-b} & \text { otherwise } \\
0 & k \leq k \leq c\end{cases} \\
\operatorname{Cr}\{\tilde{r} \geq k\}= \begin{cases}\frac{1}{2(b-a)} & a \leq k \leq b \\
\frac{2 b-r}{2(c-b)} & b \leq k \leq c \\
0 & k \geq c\end{cases}
\end{gathered}
$$

Therefore, the expected value, variance, and skewness of each triangular fuzzy variable are obtained from the equations (9), (10) and (11) [16].

$$
E(\tilde{r})=\frac{a+2 b+c}{4}
$$




$$
\operatorname{Var}(\tilde{r})= \begin{cases}\frac{33 \alpha^{3}+11 \alpha \beta^{2}+21 \alpha^{2} \beta-\beta^{3}}{384 \alpha} & \alpha>\beta \\ \frac{\alpha^{2}}{6} & \alpha=\beta \\ \frac{33 \beta^{3}+11 \alpha^{2} \beta+21 \alpha \beta^{2}-\alpha^{3}}{384 \beta} & \alpha<\beta\end{cases}
$$

Where $\alpha=b-a$ and $\beta=c-b$.

$$
S k(\tilde{r})=\frac{(c-a)^{2}}{32}[(c-b)-(b-a)]
$$

That shows if $c-b \geq b-a$, then $S k(\tilde{r}) \geq 0$ and if $c-b \leq b-a$ then $S k(\tilde{r}) \leq 0$. In particular if $S k(\tilde{r})$ is symmetric, then $b-a=c-b$ and $\operatorname{Sk}(\tilde{r})=0$. If $a=b$ then $S k(\tilde{r})$ achieves the maximum value $(c-a)^{3} / 32$ and, if $b=c$ achieves the minimum value $-(c-a)^{3} / 32[16]$.

\section{2 Liquidity risk}

Liquidity is one of the most important criteria for investors while the formation of the Portfolio. The ability of quick transaction of a huge amount of stock at a low cost and the price effect is called "liquidity". The low price effect means that asset prices have not much changed in the interval of an order to buy [17]. In fact, liquidity and liquidity risk include the main properties and microstructure factors of the market, which have threatened people's capital and play a crucial role in the investment decision to buy and sell their stocks. Illiquidity occurs when the stock price in response to low trading volumes has changed much, in fact, the lack of liquidity may have a negative impact on the stock's value [18].

Liquidity is a multi-dimensional measure, and there is no unique criterion that would cover all aspects of liquidity. Therefore, several different criteria are used that each of which represents a dimension of liquidity. So far, many measures have been introduced for liquidity, including the value of the transaction, the number of transactions, trading volume, and the difference between offered bid and buy prices $[3,17]$. Therefore liquidity of an asset cannot be accurately calculated. One of the most effective and efficient solutions is to use fuzzy numbers to express the liquidity of assets. Asset turnover rate is a factor that can reflect the liquidity of assets. Here, we assume $\mathrm{i}$-th asset turnover rate is shown by triangular fuzzy number $\tilde{l}_{i}=\left(l a_{i}, l b_{i}, l c_{i}\right)$. As a result, the portfolio turnover rate $x=\left(x_{1}, x_{2}, \ldots, x_{n}\right)$ is calculated through the equation (12).

$$
\tilde{l}(x)=\sum_{i=1}^{n} \tilde{l}_{i} x_{i}
$$

According to equation (9), the classic possibility of the mean value of the asset portfoliox $=\left(x_{1}, x_{2}, \ldots, x_{n}\right)$ turnover rate can be calculated.

$$
E\{\tilde{l}(x)\}=\sum_{i=1}^{n}\left(\frac{l a_{i}+2 l b_{i}+l c_{i}}{4}\right) x_{i}
$$

Also in this paper, possibility variance of fuzzy turnover rate is used as a measure of liquidity risk calculated based on the equation (10).

\section{3 Fuzzy multi-objective optimization model for portfolio selection}

Consider the optimal portfolio selection problem of $n$ risky assets under conditions of fuzzy uncertainty. Rates of return and turnover rates of $n$ assets, the maximum accepted risk of the portfolio and the minimum accepted turnover rate of the portfolio by investor and a maximum number of types of assets in the portfolio have been marked by triangular fuzzy numbers. Maximizing expected return on a portfolio, maximizing skewness or a chance to obtain higher expected returns and minimizing liquidity risk simultaneously, are investor's objectives. He needs for less than or equal portfolio return risk and liquidity portfolio greater than or equal to a predetermined value. On the other hand, investors intend to hold $\tilde{q}$ types of assets in their portfolios. The variables and parameters are as follows.

$\tilde{r}_{i}: \quad$ The rate of return on risky assets $i(i=1,2, \ldots, n)$, so that $\tilde{r}_{i}=\left(a_{i}, b_{i}, c_{i}\right)$ 
$\tilde{R}_{p}:$ Portfolio return rate

$\tilde{r}_{0}$ : The maximum acceptable return risk of the portfolio for the investor, so that $\tilde{r}_{0}=\left(a_{0}, b_{0}, c_{0}\right)$

$\tilde{l}_{i}: \quad$ The rate of turnover on risky assets $i(i=1,2, \ldots, n)$, so that $\tilde{l}_{i}=\left(l a_{i}, l b_{i}, l c_{i}\right)$

$\tilde{l}_{p}$ : Portfolio turnover rate

$\tilde{l}_{0}$ : The minimum acceptable turnover risk of the portfolio for the investor, so that $\tilde{l}_{0}=\left(l a_{0}, l b_{0}, l c_{0}\right)$

$x_{i}$ : Investment ratio in risky assets $i(i=1,2, \ldots, n)$

$\tilde{q}: \quad$ The maximum number of types of assets in the portfolio, so that $\tilde{q}=(q a, q b, q c)$

For modeling and formulating this problem as a mathematical expression, we suggest the following portfolio optimization model $\left(\mathrm{P}_{1}\right)$ which is based on mean-variance-skewness model.

$$
\begin{array}{ll}
\max & E\left(\tilde{R}_{p}\right)=\sum_{i=1}^{n} E\left(\tilde{r}_{i}\right) x_{i} \\
\max & \operatorname{Sk}\left(\tilde{R}_{p}\right)=\sum_{i=1}^{n} \operatorname{Sk}\left(\tilde{r}_{i}\right) x_{i} \\
\min & \operatorname{Var}\left(\tilde{l}_{p}\right)=\sum_{i=1}^{n} \operatorname{Var}\left(\tilde{l}_{i}\right) x_{i} \\
\text { s.t. } &
\end{array}
$$

$\left(\mathrm{P}_{1}\right)$

$$
\begin{aligned}
& \operatorname{Var}\left(\tilde{R}_{p}\right) \leq \tilde{r}_{0} \\
& E\left(\tilde{l}_{p}\right) \geq \tilde{l}_{0} \\
& \sum_{i=1}^{n} x_{i}=1 \\
& \sum_{i=1}^{n} \operatorname{sign}\left(x_{i}\right) \leq \tilde{q} \\
& x_{i} \geq 0, \quad i=1,2, \ldots, n
\end{aligned}
$$

The objective function (14) shows the maximization of expected portfolio returns. The objective function (15) states skewness maximization or the chance to obtain higher expected returns and the objective function (16) indicates the minimization of liquidity risk. Constrain (17) ensures that the portfolio return risk is smaller than a predetermined fuzzy number $\tilde{r}_{0}$. Constrain (18), shows that the average value of the portfolio turnover rate is not less than the proposed minimum level of $\tilde{l}_{0}$. Constrain (19), shows that the sum of assets in the portfolio is equal to one. Constrain (20), indicates that the number of assets in the portfolio must be less than or equal to the given fuzzy number $\tilde{q}$. When you want to defuzzy the fuzzy number $\tilde{q}$, any real number may be obtained, since the number of types of stocks included in the portfolio is necessarily an integer, therefore in order to overcome this problem, by using the floor function converting this number to an integer one and then it is used in modeling and solving. Constrain (21) shows reasonable limits. Using the equations (9-13) fuzzy model $\left(\mathrm{P}_{1}\right)$ has been de-fuzzy and classical model $\left(\mathrm{P}_{2}\right)$ is obtained as follows.

$$
\max E\left(\tilde{R}_{p}\right)=\sum_{i=1}^{n}\left(\frac{a_{i}+2 b_{i}+c_{i}}{4}\right) x_{i}
$$

$$
\max \operatorname{Sk}\left(\tilde{R}_{p}\right)=\sum_{i=1}^{n}\left(\frac{\left(c_{i}-a_{i}\right)^{2}}{32}\left[\left(c_{i}-b_{i}\right)-\left(b_{i}-a_{i}\right)\right]\right) x_{i}
$$




$$
\begin{aligned}
& \min \operatorname{Var}\left(\tilde{l}_{p}\right)=\frac{11\left(\sum_{i=1}^{n}\left(l c_{i}-l a_{i}\right) x_{i}\right)^{2}\left|\sum_{i=1}^{n}\left(2 l b_{i}-l a_{i}-l c_{i}\right) x_{i}\right|}{192\left(\sum_{i=1}^{n}\left(l c_{i}-l a_{i}\right) x_{i}+\left|\sum_{i=1}^{n}\left(2 l b_{i}-l a_{i}-l c_{i}\right) x_{i}\right|\right)} \\
& +\frac{\left.2\left(8 \sum_{i=1}^{n}\left(l c_{i}-l a_{i}\right) x_{i}\right)+3\left|\sum_{i=1}^{n}\left(2 l b_{i}-l a_{i}-l c_{i}\right) x_{i}\right|\right)\left(\left(\sum_{i=1}^{n}\left(l c_{i}-l b_{i}\right) x_{i}\right)^{2}+\left(\sum_{i=1}^{n}\left(l b_{i}-l a_{i}\right) x_{i}\right)^{2}\right)}{192\left(\sum_{i=1}^{n}\left(l c_{i}-l a_{i}\right) x_{i}+\left|\sum_{i=1}^{n}\left(2 l b_{i}-l a_{i}-l c_{i}\right) x_{i}\right|\right)}
\end{aligned}
$$

s.t.

$$
\begin{aligned}
& \frac{\left.2\left(8 \sum_{i=1}^{n}\left(c_{i}-a_{i}\right) x_{i}\right)+3\left|\sum_{i=1}^{n}\left(2 b_{i}-a_{i}-c_{i}\right) x_{i}\right|\right)\left(\left(\sum_{i=1}^{n}\left(c_{i}-b_{i}\right) x_{i}\right)^{2}+\left(\sum_{i=1}^{n}\left(b_{i}-a_{i}\right) x_{i}\right)^{2}\right)}{192\left(\sum_{i=1}^{n}\left(c_{i}-a_{i}\right) x_{i}+\left|\sum_{i=1}^{n}\left(2 b_{i}-a_{i}-c_{i}\right) x_{i}\right|\right)} \\
& +\frac{11\left(\sum_{i=1}^{n}\left(c_{i}-a_{i}\right) x_{i}\right)^{2}\left|\sum_{i=1}^{n}\left(2 b_{i}-a_{i}-c_{i}\right) x_{i}\right|}{192\left(\sum_{i=1}^{n}\left(c_{i}-a_{i}\right) x_{i}+\left|\sum_{i=1}^{n}\left(2 b_{i}-a_{i}-c_{i}\right) x_{i}\right|\right)} \leq \frac{a_{0}+4 b_{0}+c_{0}}{6} \\
& \sum_{i=1}^{n}\left(\frac{l a_{i}+2 l b_{i}+l c_{i}}{4}\right) x_{i} \geq \frac{l a_{0}+4 l b_{0}+l c_{0}}{6} \\
& \sum_{i=1}^{n} x_{i}=1 \\
& \sum_{i=1}^{n} \operatorname{sign}\left(x_{i}\right) \leq\left[\frac{q a+4 q b+q c}{6}\right] \\
& x_{i} \geq 0, \quad i=1,2, \ldots, n
\end{aligned}
$$

\section{Solution Methodology}

To solve the portfolio selection optimization problem in the primary studies, the exact methods such as linear programming, nonlinear programming, and goal programming have been used. But since it has been proven that portfolio selection optimization problem such $\mathrm{P}_{2}$ model has a high complexity, achieving optimal solution for the problem with large dimensions and even medium in a reasonable time is not possible $[3,5,6]$. Thus many attempts have been performed to obtain near-optimal and high quality solutions by employing heuristic and meta-heuristic methods instead of exact methods [6,9]. One of meta-heuristic methods that properly has coped with solving this kind of problem is genetic

\begin{tabular}{|c|c|c|c|c|c|c|c|c|c|c|}
\hline$C_{k}=$ & 0.06 & 0.14 & 0 & 0 & 0.35 & 0.21 & 0.19 & 0.18 & 0 & 0.64 \\
\hline
\end{tabular}
algorithm [13,14]. Meta-heuristic NSGA-II algorithm is one of the most versatile and powerful genetic algorithms for solving multi-objective optimization problems and its efficiency in solving various problems has been proven. This algorithm was introduced in 2000 by Deb et al. [13]. In this paper, in order to solve the multi-objective portfolio selection problem optimization $\mathrm{P}_{2}$ that its model was presented in the previous section, a multi-objective NSGA-II algorithm have developed as follows.

\section{1 Representation of chromosome and generation of initial population}

On the problem of optimal portfolio selection, a solution $x=\left(x_{1}, x_{2}, \ldots, x_{n}\right)$ can be represented by chromosome $c=\left(c_{1}, c_{2}, \ldots, c_{n}\right)$, so that genes $c_{j}$ are restricted in the range of $[0,1]$. For example k-th chromosome will be as follows. 
The relationship between feasible solution and chromosome can be presented as the expression (30), ensuring that $\sum_{j=1}^{n} x_{j}=1$ is always true.

$$
x_{j}=\frac{c_{j}}{c_{1}+c_{2}+\ldots+c_{n}} \quad, \quad j=1,2, \ldots, n
$$

According to equation (30), the solution associated with chromosome $C_{k}$ is as follows.

\begin{tabular}{|c|c|c|c|c|c|c|c|c|c|c|}
\hline$x_{k}=$ & 0.034 & 0.079 & 0 & 0 & 0.200 & 0.119 & 0.107 & 0.100 & 0 & 0.361 \\
\hline
\end{tabular}

The initial population size is considered equal to an integer and the above-mentioned process will be repeated with the number until the initial population will be produced and they will be indicated by the notation or $C_{1}, C_{2}, \ldots, C_{p o p-s i z e}$.

\section{2 Handling the constrain of maximum number of types of stocks in the portfolio}

To satisfy constrain of maximum number of types of stocks in the portfolio (constrain 28), a random number less than or equal $k$ is generated and is called $t$. Then, if the number of genes higher than zero in the chromosome is larger than $t$, we hold $t$ numbers of the largest values of $x_{j}$ and put the other $x_{j}$ 's zero. In the next step, to modify chromosomes and meet the constrain (27), again the equation (30) is used and normalization of genes will be conducted. For example, if the number of genes of the chromosome is $n=10$, the maximum number of types of stocks in the portfolio is $k=6$, then $t=5$ will be achieved. Chromosome $C_{k}$ presented in the section above is modified as follows, which is called $C_{k}^{\prime}$. The solution associated with the chromosome $C_{k}^{\prime}$, has been normalized according to equation (30), and it is called $x_{k}^{\prime}$.

\begin{tabular}{|c|c|c|c|c|c|c|c|c|c|c|}
\hline$C_{k}^{\prime}=$ & 0 & 0 & 0 & 0 & 0.35 & 0.21 & 0.19 & 0.18 & 0 & 0.64 \\
\hline$x_{k}^{\prime}=$ & 0 & 0 & 0 & 0 & 0.223 & 0.134 & 0.121 & 0.114 & 0 & 0.408 \\
\hline
\end{tabular}

\section{3 Crossover and mutation operators}

Many famous crossover operators for chromosomes in the optimal portfolio selection problem are available [3]. We use single-point crossover operator. First, two chromosomes are selected as parents. A random number between 1 and $n-1$ has been generated to determine the crossing point of two chromosomes. Then two child chromosomes are generated by moving genes of two parent chromosomes from the crossing point.

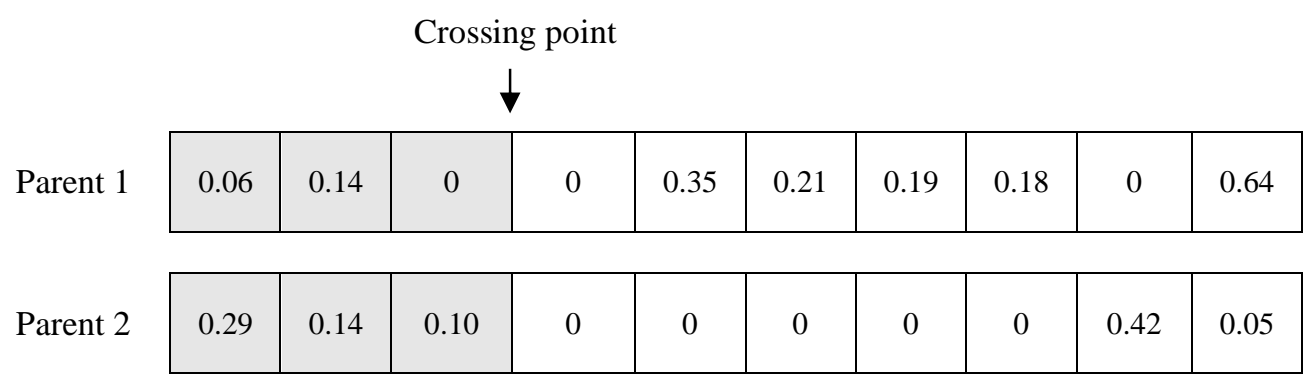

After applying single-point crossover operator

\begin{tabular}{|c|c|c|c|c|c|c|c|c|c|c|}
\hline Child 1 & 0.06 & 0.14 & 0 & 0 & 0 & 0 & 0 & 0 & 0.42 & 0.05 \\
\hline
\end{tabular}




\begin{tabular}{|l|l|l|l|l|l|l|l|l|l|}
\hline 0.29 & 0.14 & 0.10 & 0 & 0.35 & 0.21 & 0.19 & 0.18 & 0 & 0.64 \\
\hline
\end{tabular}

After the generation of child chromosomes, due to constrain of the maximum number of types of stocks in the portfolio and the equation (30), they will be normalized and modified if needed to have chromosomes that are valid. If, for example, the maximum number of types of stocks included in the portfolio are $k=6$ and $t=5$, we have:

After modification and validation

Child 1

\[ \]
\begin{tabular}{|l|c|c|c|c|c|c|c|c|c|}
\hline 0.089 & 0.209 & 0 & 0 & 0 & 0 & 0 & 0 & 0.627 & 0.075 \\
\hline 0.176 & 0 & 0 & 0 & 0.212 & 0 & 0.115 & 0.109 & 0 & 0.388 \\
\hline
\end{tabular}

To carry mutations in chromosomes, first two genes from chromosomes are randomly selected and their values are exchanged with each other, such as the following example:

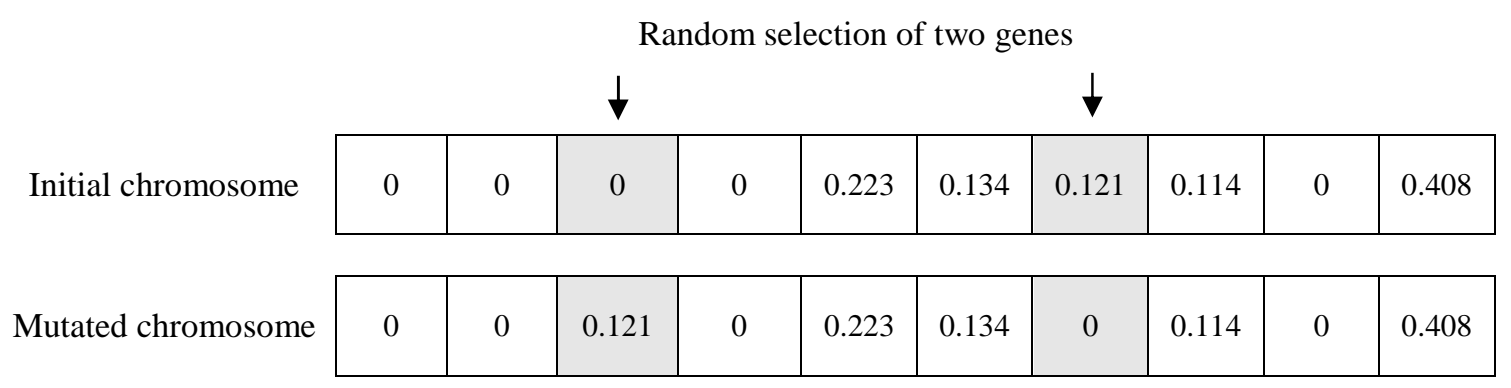

\section{4 Evaluation of the solutions based on NSGA-II algorithm}

In the NSGA-II algorithm, first, the number of children $\left(Q_{t}\right)$ can be built, using the parental population $\left(P_{t}\right)$, respectively using crossover and mutation operators. Two parents and children populations have been combined with each other and create the population $R_{t}$ with the size of $2 N$. Then the fast non-dominated sorting approach will be used to categorize the entire population $R_{t}$. According to this approach, the solutions will be put in different categories with different non-dominated fronts. The total number of categories has been shown with $k$. All solutions in categories or the first front $\left(F_{l}\right)$ are the best non-dominated solutions of the population and the worst solutions of the population will be in the last front $\left(F_{k}\right)$. It is clear that in terms of the value of fitness, the most fitness will be assigned to the solutions of the first front population and this fitness will be decreased in the next fronts so that, the lowest fitness will be assigned to the solutions of the last front. In Fig. 2., the image of categorization of the solution based on the fast non-dominated sorting approach for the problem with two-objective minimization has been shown [19].

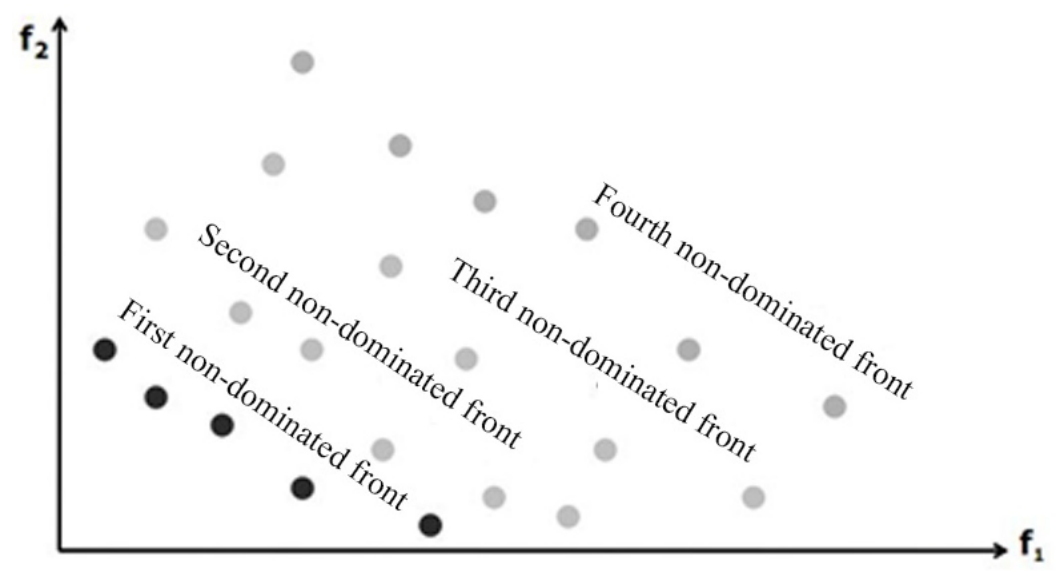


Fig. 2: Fast non-dominated sorting approach

In the next step, based on sorting solutions, the next generation will be filled with these fronts. Filling the next generation $\left(P_{t}+1\right)$ has been started with the best non-dominated front and then respectively with the second non-dominated front and the third and so on, it continues until $P_{t}+1$ would be filled. Since the size is equal to $2 N$ all members cannot be placed in $P_{t}+1$ and the remaining solutions will be easily deleted. Fig. 3. shows the performance of the algorithm NSGAII [19].

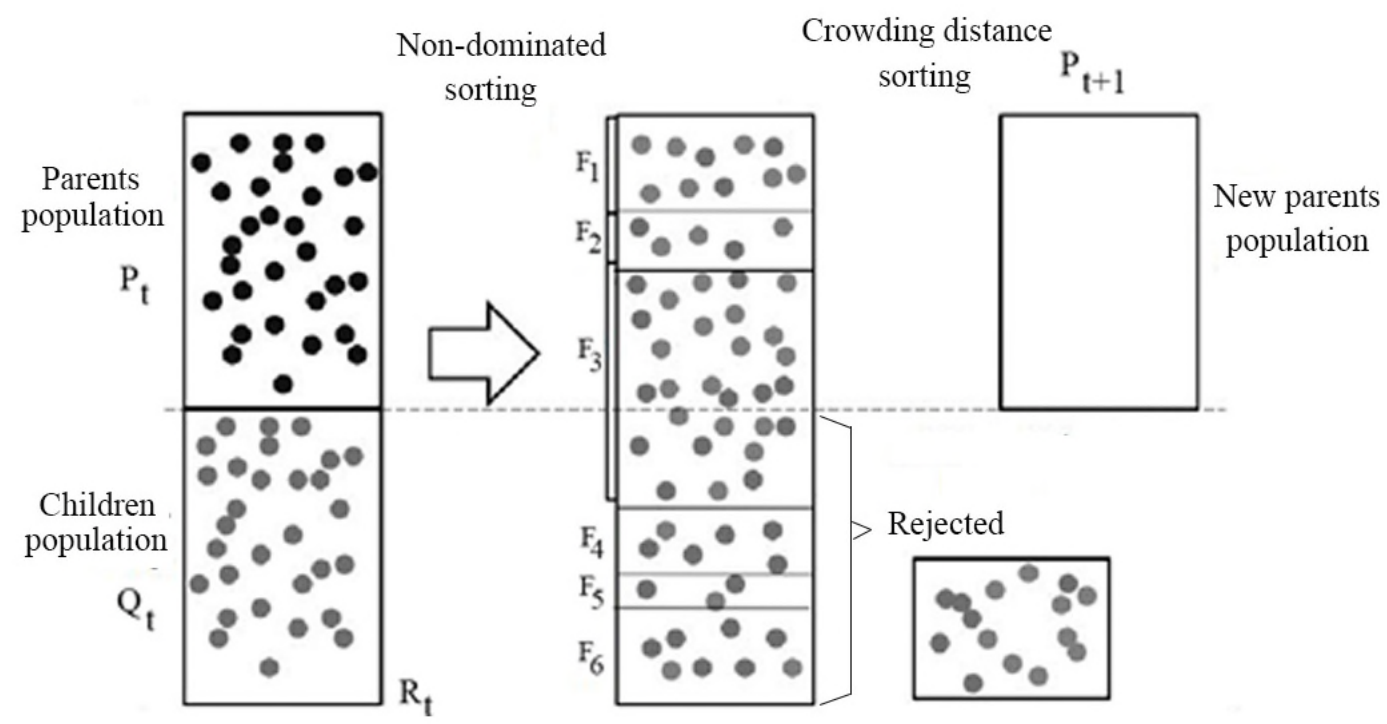

Fig. 3: Schema of performance of the algorithm NSGA-II

In regard of the solutions eliminated in the last front by the elitism operator more skills should be used and the solutions in the area with less crowded should be maintained. In fact, to observe the principle of the density of the solutions, the solutions which are in smaller crowding area have priority to fill $P_{t}+1$.

\section{Computational Results}

In order to evaluate the performance of the model and the proposed solution, random portfolios were selected from among the top 50 companies in Tehran Stock Exchange in the fourth quarter of 2015. The triangular distribution function of turnover and return rates have been obtained for 50 companies chosen through historical data from financial software Rahavard Novin during 2014 to 2015. Maximum of types of stocks in the portfolio selected was considered as a random fuzzy number $(\tilde{q})$ that, the amount of which is determined by investors. Problems were classified into two groups with small and large size. Problems with the maximum number of 10 types of stocks were considered as small-scale problems and with a minimum of 15 types of stocks were considered as large-scale problems.

In order to compare and evaluate the performance of the proposed solution method, two VEGA and NSGA algorithms were also developed. The above-mentioned three algorithms have been coded with MATLAB (R2014a) software. To run the program coded, a personal computer with a $2.3 \mathrm{GHz}$ seven core processor and 8 GB main memory has been used. In order to ensure a fair comparison among the three algorithms developed, their parameters were set identically. The initial population size is 100 and crossover and mutation rates are respectively 0.1 and 0.8 . Number of generations produced as a condition for stopping the algorithm was applied and were set as 2,000. For each of the problems produced, each algorithm was run five times and the results are expressed as the average of the value obtained.

There are numerous and diverse indicators to compare and evaluate the performance of multi-objective metaheuristic algorithms. In this paper two useful indices of the quality and dispersion have been used for comparison. Quality index compares the quality of the Pareto solutions obtained by each method. To calculate the quality index, all solutions obtained by all three methods has been levelled together and it is specified that how many percent is assigned to each method [19]. The higher the percentage, the higher quality algorithm has. Dispersion index is used for determining the non-dominated solutions found on the optimal frontier. Dispersion index is defined as follows:

$$
D=\sqrt{\sum_{i=1}^{N} \max \left(\left\|x_{t}^{i}-y_{t}^{i}\right\|\right)}
$$


In equation (31), $\left\|x_{t}^{i}-y_{t}^{i}\right\|$ represents the Euclidean distance between the two solutions of $x_{t}^{i}$ and $y_{t}^{i}$ on the optimal frontier. Test results for small-scale problems are provided in Table 1 and for large-scale problems in Table 2 [19].

Table 1: Comparison of results obtained for small-scale problems

\begin{tabular}{|c|c|c|c|c|c|c|c|c|}
\hline \multirow[b]{2}{*}{$\begin{array}{c}\text { Problem } \\
\text { No. }\end{array}$} & \multicolumn{2}{|c|}{ Problem information } & \multicolumn{3}{|c|}{ Quality index } & \multicolumn{3}{|c|}{ Dispersion index } \\
\hline & $\begin{array}{l}\text { Number } \\
\text { of types } \\
\text { of stocks }\end{array}$ & $\begin{array}{l}\text { The maximum number } \\
\text { of types of stocks in } \\
\text { the portfolio }\end{array}$ & VEGA & NSGA & NSGA-II & VEGA & NSGA & NSGA-II \\
\hline 1 & 8 & $(2,4,5)$ & 13.12 & 14.51 & 72.37 & 327.4 & 471.1 & 654.3 \\
\hline 2 & 8 & $(4,5,7)$ & 18.15 & 26.04 & 55.81 & 294.1 & 529.3 & 608.9 \\
\hline 3 & 8 & $(3,5,6)$ & 5.76 & 17.11 & 77.13 & 309.7 & 435.1 & 634.4 \\
\hline 4 & 9 & $(2,3,6)$ & 9.46 & 4.12 & 86.42 & 169.3 & 376.9 & 691.6 \\
\hline 5 & 9 & $(3,5,7)$ & 21.49 & 17.06 & 61.45 & 371.8 & 443.6 & 537.1 \\
\hline 6 & 9 & $(4,5,6)$ & 0 & 5.41 & 94.59 & 287.9 & 367.4 & 647.5 \\
\hline 7 & 10 & $(4,5,7)$ & 1.06 & 11.43 & 87.51 & 297.3 & 638.2 & 671.6 \\
\hline 8 & 10 & $(3,4,8)$ & 0 & 0 & 100 & 301.6 & 543.7 & 656.4 \\
\hline 9 & 10 & $(5,5,7)$ & 0 & 5.86 & 94.14 & 249.2 & 426.3 & 716.7 \\
\hline 10 & 10 & $(4,6,6)$ & 6.42 & 21.09 & 72.49 & 341.2 & 481.5 & 653.9 \\
\hline
\end{tabular}

Table 2. Comparison of the results obtained for large-scale problems

\begin{tabular}{|c|c|c|c|c|c|c|c|c|}
\hline \multirow[b]{2}{*}{$\begin{array}{c}\text { Problem } \\
\text { No. }\end{array}$} & \multicolumn{2}{|c|}{ Problem information } & \multicolumn{3}{|c|}{ Quality index } & \multicolumn{3}{|c|}{ Dispersion index } \\
\hline & $\begin{array}{l}\text { Number } \\
\text { of types } \\
\text { of stocks }\end{array}$ & $\begin{array}{l}\text { The maximum number } \\
\text { of types of stocks in } \\
\text { the portfolio }\end{array}$ & VEGA & NSGA & NSGA-II & VEGA & NSGA & NSGA-II \\
\hline 1 & 20 & $(6,9,10)$ & 0 & 4.84 & 95.16 & 1905.4 & 8943.7 & 6157.2 \\
\hline 2 & 20 & $(12,15,17)$ & 5.81 & 6.57 & 87.62 & 9462.3 & 7953.1 & 13462 \\
\hline 3 & 20 & $(5,7,8)$ & 0 & 0 & 100 & 6142.7 & 9126.4 & 11482 \\
\hline 4 & 30 & $(12,14,14)$ & 4.14 & 15.17 & 80.69 & 7345.9 & 8967.9 & 10694 \\
\hline 5 & 30 & $(8,8,9)$ & 8.47 & 15.08 & 76.45 & 5849.5 & 6128.4 & 9652.8 \\
\hline 6 & 30 & $(11,12,15)$ & 0 & 1.71 & 98.29 & 9416.4 & 6455.3 & 11653 \\
\hline 7 & 30 & $(10,10,10)$ & 0 & 0 & 100 & 4391.2 & 7664.8 & 10264 \\
\hline 8 & 30 & $(15,16,20)$ & 1.13 & 2.73 & 96.14 & 8438.6 & 9749.1 & 11946 \\
\hline
\end{tabular}

The results of carrying out tests prove that the proposed model and developed NSGA-II algorithm have a high performance for modeling and solving the multi-objective optimization portfolio problem under fuzzy uncertainty conditions. The proposed solution method based on NSGA-II algorithm has priority to produce Pareto solutions, according to both the quality and dispersion indices in the small and large-scale problems in comparison with VEGA and NSGA algorithms.

\section{Computational Results}

In this paper, a fuzzy multi-objective programming model based on mean-variance-skewness model was developed for optimal portfolio selection that includes less simplifying assumptions than the other available models. The proposed model includes three objective functions of maximizing expected return, maximizing skewness or the chance to gain higher expected returns and minimizing liquidity risk. In this model, rates of return, rates of turnover, and the maximum number of types of stocks included in the portfolio were considered triangular fuzzy numbers. To solve this problem an elitist non-dominated sorting genetic algorithm (NSGA-II) was developed. To compare and evaluate the performance of the model and the proposed solution, the top 50 companies in Tehran Stock Exchange in the fourth quarter of 2015 were considered and based on historical data, the triangular distribution function of expected rates of return and financial turnover rates are obtained. Proposed solution method with two vector evaluation genetic algorithm (VEGA) and nondominated sorting genetic algorithm (NSGA) was compared and analyzed based on two quality and dispersion indicators in small and large-scale problems. The results show that the proposed solution based on NSGA-II algorithm has the very acceptable performance for the production of Pareto solutions, with high quality and variety and a higher dispersion than the VEGA and NSGA algorithms. In other words, the proposed method gives the more optimal portfolio to the investors. 
Considering the cost of transactions and other types of risk in modeling as well as developing innovative hybrid algorithms to solve the problem for future research are recommended.

\section{REFERENCES}

[1] Nabavi Chashemi, A., Yousefi KarChangi, R. (2011). Determination of the optimal portfolio using fuzzy goal programming technique. Journal of Financial Engineering and Management Securities, vol. 9, p. 107-130. (In Persian)

[2] Vafaei, F., Letafati, S., Ardalan, A. (2012). Designing portfolio optimization model using fuzzy mathematical programming (Case Study: Bank Melli Iran Investment Company). Journal of Industrial Management Faculty of Humanities, Islamic Azad University of Sanandaj, vol. 7, no. 21, p. 1-9. (In Persian)

[3] Liu, Y.J., Zhang, W.G. (2013). Fuzzy portfolio optimization model under real constraints. Insurance: Mathematics and Economic, vol. 53, no. 3, p. 704-711.

[4] Alinezhad, A., Zohrehbandian, M., Kian, M., Ekhtiari, M., Esfandiari, N. (2011). Extension of Portfolio Selection Problem with Fuzzy Goal Programming: A Fuzzy Allocated Portfolio Approach. Journal of Optimization in Industrial Engineering, vol. 4, no. 9, p. 69-76.

[5] Shahmohammadi, M., Emami, L., Zare Mehrjerdi, Y. (2012). A hybrid intelligent algorithm for portfolio selection using fuzzy mean-variance-skewness. International Journal of Industrial Engineering \& Production Management, vol. 4, no. 23, p. 448-458. (In Persian)

[6] Li, X., Qin, Z., Kar, S. (2010). Mean-variance-skewness model for portfolio selection with fuzzy returns. European Journal of Operational Research, vol. 202, no. 1, p. 239-247.

[7] Ghahtarani, A.R., Najafi, A.A. (2013). A Fuzzy Approach to Mean-CDaR Portfolio Optimization. International Journal of Applied Operational Research, vol. 3, no. 3, p. 95-104.

[8] Khalifa, H.A., ZeinEldin, R.A. (2014). Fuzzy programming approach for portfolio selection problems with fuzzy coeffiecents. International Journal of Scientific Knowledge, vol. 4, no. 7, p. 40-47.

[9] Zhou, R., Zhan, Y., Cai, R., Tong, G. (2015). A Mean-Variance Hybrid-Entropy Model for Portfolio Selection with Fuzzy Returns. Entropy, vol. 17, no. 5, p. 3319-3331.

[10] Ammar, E., Khalifa, H.A. (2003). Fuzzy portfolio optimization a quadratic programming approach. Chaos, Solitons \& Fractals, vol. 18, no. 5, p. 1045-1054.

[11] Huang, X. (2007). A new perspective for optimal portfolio selection with random fuzzy returns. Information Sciences, vol. 177, no. 23, p. 5404-5414.

[12] Gupta, P., Mehlawat, M.K., Saxena, A. (2008). Asset portfolio optimization using fuzzy mathematical programming. Information Sciences, vol. 178, no. 6, p. 1734-1755.

[13] Shibt Al-Hamdi, S.A., Hemmati, M., Esfandiar, M. (2014). Application of multi-objective genetic algorithms (NSGAII) in the optimal portfolio selection of Stock Exchange. Journal of Management, vol. 11, no. 34, p. 21-34. (In Persian)

[14] Delvi, M.R., Baghi, A., Abdolbaghi, A., Kazemi, J. (2015). Application of genetic algorithms for multi-objective optimization in bank facilities portfolios (Case Study of Melli bank facilities in Isfahan province). Accounting and Auditing Research, vol. 7, no. 27, p. 100-115. (In Persian)

[15] Liu, B. and Liu, Y.K. (2002). Expected Value of Fuzzy Variable and Fuzzy Expected Value Models. IEEE Transactions on Fuzzy Systems, vol. 10, no. 4, p. 445-450.

[16] Wang, Z. and Tian, F. (2010). A Note of the Expected Value and Variance of Fuzzy Variables. International Journal of Nonlinear Science, vol. 9, no. 4, p. 486-492.

[17] Eslami Bidgoli, G., Sarenj, a. (2008). portfolio selection Using three measures of mean return, the standard deviation of returns and liquidity in the Tehran Stock Exchange. Accounting and auditing studies, vol. 15, no. 53, p. 3-16. (In Persian)

[18] Hydari, S.A., Fallah Shams, M.F., Hashemi N. (2011). Studying the relationship between liquidity risk and price in in the Tehran Stock Exchange. Journal of Financial Engineering and Management Securities, vol. 2, no. 9, p. 207229. (In Persian)

[19] Lwin, K., Qu, R., Kendall, G. (2014). A learning-guided multi-objective evolutionary algorithm for constrained portfolio optimization. Applied Soft Computing, vol. 24, p. 757-772. 